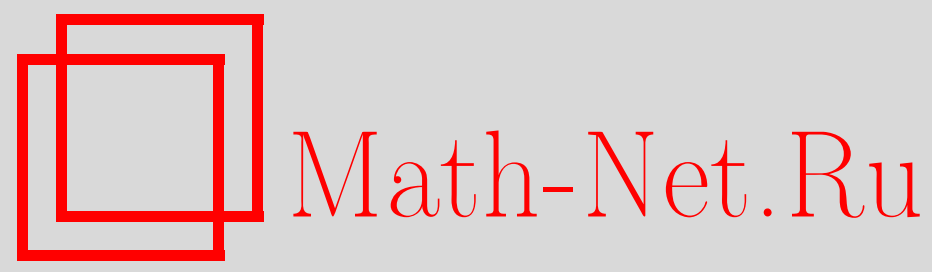

А. А. Туганбаев, Дистрибутивные и мультипликационные модули и кольца, Матем. заметки, 2004, том 75, выпуск $3,425-434$

DOI: https://doi.org/10.4213/mzm46

Использование Общероссийского математического портала Math-Net.Ru подразумевает, что вы прочитали и согласны с пользовательским соглашением http://www.mathnet.ru/rus/agreement

Параметры загрузки:

IP: 34.229 .45 .116

26 апреля 2023 г., 12:29:08 


\title{
ДИСТРИБУТИВНЫЕ И МУЛЬТИПЛИКАЦИОННЫЕ МОДУЛИ И КОЛЬЦА
}

\author{
А. А. Туганбаев
}

\begin{abstract}
Изучаются кольца, в которых каждый идеал является конечно порожденным мультипликационньм правым идеалом.

Библиограффия: 8 названий.
\end{abstract}

Все кольца ассоциативны и с ненулевой единицей. Выражения типа "нетерово кольцо" означают, что соответствуюшие условия вьполнены справа и слева. Через Lat $(M)$ обозначается решетка всех подмодулей модуля $M$. Через $\operatorname{Lat}\left(A_{A}\right)$ и $\operatorname{Lat}\left({ }_{A} A_{A}\right)$ обозначаются решетка всех правых идеалов и решетка всех идеалов кольца $A$. Модуль $M$ назьвается дистрибутивным (или, для краткости, $d$-модулем), если Lat $(M)$ дистрибутивна. Модуль $M_{A}$ назьвается мультипликационным (или, для краткости, $m$-модулем), если для каждого его подмодуля $N$ существует такой идеал $B$ кольца $A$, что $N=$ $M B$. Правый (левьй) идеал $B$ кольца $A$ назьвается мультипликационнылм правым

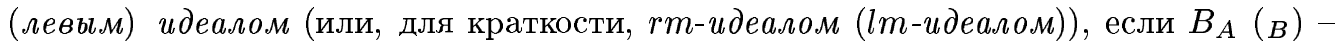
$m$-модуль. Идеал, являющийся $r m$-идеалом и $l m$-идеалом, назьвается $m$ - $u$ деалом. Ясно, что $M_{A}-m$-модуль $\Longleftrightarrow M(N: M)=N$ для любого $N \in \operatorname{Lat}(M) \Longleftrightarrow M r(M / N)=$ $N$ для любого $N \in \operatorname{Lat}(M)$. (Для любых подмножеств $X, Y \subseteq M_{A}$ через $(Y: X)$ обозначается множество $\{a \in A \mid X a \subseteq Y\} \subseteq A$; в частности, $r(X)=(0: X)$ - правьй аннулятор для $X$ в $A$.) В общем случае класс $m$-модулей не сравним по включению с классом $d$-модулей. Любая квазициклическая абелева групша является немультипликационным $d$-модулем над $\mathbb{Z}$. Если $A=k[x, y]$ - кольцо многочленов от двух переменных $x, y$ над полем $k$, то $A_{A}$ - недистрибутивный $m$-модуль, поскольку $(x+y) A \cap$ $(x A+y A) \neq(x+y) A \cap x A+(x+y) A \cap y A$. Над коммутативным кольцом класс всех $m$-модулей содержит все проективные идеалы [1], все циклические модули (см. лемму 4), все конечно порожденные $d$-модули (см. [2]) и все идеалы, порожденные идемпотентами (см. [3, с. 757] или лемму 6(7)). О m-модулях над коммутативньпи кольцами см., например, [1]-[6]. В [7] и [8] затрагиваются $m$-модули над не обязательно коммутативными кольцами. Основные результаты работы- теоремы А и В.

Теорема А. Для кольиа А равносильны следующие условия:

(1) в А каждый правый идеал - конечно порожденный rт-идеал;

(2) A - нетерово справа кольцо и каждый его первичный идеал это rm-идеал;

(3) A- дистрибутивное справа нетерово справа кольцо;

Работа выполнена при поддержке Российского фонда фундаментальных исследований. 
(4) А инвариантно справа и каждый правый $m$-модуль - нетеров $d$-модуль;

(5) в $A$ каждый правый идеал $M$ является таким rm-идеалом, что $M \neq M P$ для любого максимального идеала $P$ кольиа $A$, содержащего $r(M)$.

Теорема В. (1) Если $A$ - кольио с коммутативным умнохсением первичных

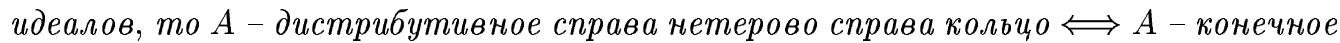
прямое произведение артиновых справа цепных справа колеи и таких инвариантных справа областей $D_{i}$, что для каждой $D_{i}$ любое собственное фактор-кольцо области $D_{i}$ является конечным прямым произведением артиновых справа чепных справа колеи.

(2) Пусть кольио $A$ либо не содержит бесконечных прямых сумм ненулевых идеалов, либо имеет лишь конечное число минимальных первичных идеалов. $B$ кольие $A$ все правые идеаль - rm-идеаль и все левые идеаль -rm-идеалы $\Longleftrightarrow в$ кольие $A$ все полупервичные идеаль $-m$-идеалы $\Longleftrightarrow A-$ конечное прямое произведение инвариантных артиновых локальных колеи, главных идеалов и инвариантных нетеровых областей, у которых все ненулевые идеалы являются обратимыми .

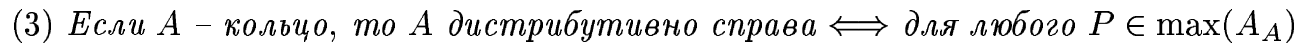
любые два $P$-замкнутых правых идеала кольца $A$ сравнимы по включению.

Доказательства теорем А и В разбиты на ряд утверждений. Приведем необходимые обозначения и определения. Для модуля $M$ через $J(M), \operatorname{End}(M)$ и $\max (M)$ обозначаются его радикал Джекобсона, кольцо эндоморфизмов и множество всех максимальных подмодулей соответственно. Для кольца $A$ через $\max \left({ }_{A} A_{A}\right)$ (соответственно, $\left.\operatorname{Lat}\left({ }_{A} A_{A}\right), \bmod (A), S(A)\right)$ обозначается множество всех его максимальных идеалов (соответственно, решетка всех идеалов, категория всех правых $A$-модулей и множество всех неделителей нуля). Кольцо $A$ назьвается регулярным, если $a \in a A a$ для каждого $a \in A$. Кольцо назьвается инвариантным справа, если все его правые идеалы являются идеалами. Кольцо называется полупервичным (редуцированным), если оно не имеет ненулевых нильпотентных идеалов (элементов). Идеал $P$ кольца $A$ назьвается первичным (вполне первичным), если произведение двух идеалов (элементов) кольца $A$ лежит в $P$ только в случае, если хотя бы один из этих идеалов (элементов) лежит в $P$. Ясно, что все вполне первичные идеалы первичны и $A-$ вполне первичньй идеал. Кольцо назьвается почти иелостным, если оно обладает нильпотентньп вполне первичным идеалом. Подмножество $S$ кольца $A$ назьвается перестановочныл справа, если для любых $a \in A$ и $s \in S$ существуют такие $b \in A$ и $t \in S$, что $a t=s b$. Модуль называется равномерным, если в нем любые два ненулевых подмодуля имеют ненулевое пересечение. Пусть $A$ - кольцо, $P \in \max \left(A_{A}\right), M \in \bmod (A)$ и $X \subseteq M$. Множество $X$ назьвается $P$-замкнутым (в $M$ ), если $X$ содержит любой такой элемент $m \in M$, что $m a \in X$ для некоторого $a \in A \backslash P$. Через $C_{P}(M)$ обозначается множество всех $P$-замкнутых подмножеств в $M$. Если $Y \subseteq M$, то пересечение всех $P$-замкнутых подмножеств в $M$, содержащих $Y$, является $P$-замкнутьм и называется $P$-замыканием для $Y$ в $M$.

Лемма 1. Пусть $M-$ правый $m$-модуль над кольиом $A$. Тогда

(1) каждый фактор-модуль $M / N-m$-модуль;

(2) если $N \in \operatorname{Lat}(M)$ и $N \cap M B=N B$ для кажсдого идеала $B$ кольиа $A$, то $N$ - т-модуль; 
(3) если $M \neq M P$ для каждого такого $P \in \max \left({ }_{A} A_{A}\right)$, ито $r(M) \subseteq P$, то $M$ конечно порожден;

(4) если А регулярно, то каждый $N \in \operatorname{Lat}(M)$ - дистрибутивный $m$-модуль;

(5) если $A$ - кольио с условием максимальности для идеалов, то $M$ - нетеров модуль;

(6) если $M B \cap M C=M(B \cap C)$ для любых идеалов $B$ и $C$ кольи а $A$, то отображение $\varphi: B \rightarrow M B$ является сюрдективным решеточным гомоморфизмом из решетки $\operatorname{Lat}\left({ }_{A} A_{A}\right)$ на решетку $\operatorname{Lat}(M)$.

ДоказАтельство. (1) Пусть $X \in \operatorname{Lat}(M)$ и $N \subseteq X$. Так как $X=M B$ для некоторого идеала $B$, то $X / N=(M / N) B$.

(2) Пусть $X \in \operatorname{Lat}(N)$. Так как $X=M B$ для некоторого идеала $B$, то $X=N \cap$ $M B=N B$.

(3) Пусть $\left\{P_{i}\right\}_{i \in I}$ - множество всех максимальных идеалов в $A$, содержаших $r(M)$. По условию $M \neq M P_{i}$ для каждого $P_{i}$. Для каждого $i \in I$ существует такой циклический $X_{i} \in \operatorname{Lat}(M)$, что $X_{i} \not \subset M P_{i}$. Обозначим через $B$ идеал $\sum_{i \in I} r\left(M / X_{i}\right)$. Тогда $B \supseteq r\left(M / X_{i}\right) \supseteq r(M)$. Допустим, что $A=B$. Тогда существует такоеконечноеподмножество $J$ в $I$, что $A=\sum_{j \in J} r\left(M / X_{j}\right)$. Так как $M-m$-модуль, то $X_{j}=M r\left(M / X_{j}\right)$ для каждого $j \in J$. Поэтому $M=M A=M\left(\sum_{j \in J} r\left(M / X_{j}\right)\right)=\left(\sum_{j \in J} M r\left(M / X_{j}\right)\right)=$ $\sum_{j \in J} X_{j}$. Так как $M=\sum_{j \in J} X_{j}$, то $M$ конечно порожден. Допустим, что $A \neq B$. Существует такой $P \in \max \left({ }_{A} A_{A}\right)$, что $P \supseteq B \supseteq r(M)$. Тогда $P=P_{i}$ для некоторого $i \in I$ и $r\left(M / X_{i}\right) \subseteq B \subseteq P_{i}$. Так как $M-m$-модуль, то $X_{i}=M r\left(M / X_{i}\right) \subseteq M P_{i}$; получили противоречие.

(4) Пусть $B$ - идеал в $A$ и $x \in N \cap M B$. Тогда $x=\sum_{i=1}^{n} m_{i} b_{i}$ для некоторых $m_{1}, \ldots, m_{n} \in M$ и $b_{1}, \ldots, b_{n} \in B$. Так как $A$ регулярно, его конечно порожденньй левый идеал $C=\sum_{i=1}^{n} A b_{i}$ порождается некоторым идемпотентом $e$. Пусть $b_{i}=a_{i} e$, где $a_{i} \in A, i=1, \ldots, n$. Если $m=\sum_{i=1}^{n} m_{i} a_{i}$, то $x=m e \in N$ и $x=x e \in N B$. Поэтому $N \cap M B \subseteq N B \subseteq N \cap M B$. По (2) $N-m$-модуль. Поэтому в $M$ все подмодули - $m$-модули. По (3) $M-d$-модуль. Поэтому $N-d$-модуль.

(5) Допустим, что Lat $(M)$ содержит бесконечную строго возрастающую цепь $X_{1} \subset$ $X_{2} \subset \cdots$. Так как $X_{i}=M\left(X_{i}: M\right)$ для всех $i$, то $\operatorname{Lat}\left({ }_{A} A_{A}\right)$ содержит бесконечную строго возрастающую цепь $\left(X_{1}: M\right) \subset\left(X_{2}: M\right) \subset \cdots$.

(6) Утверждение проверяется непосредственно.

Лемма 2. Для кольиа А равносильны следующие условия:

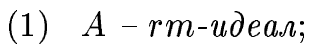

(2) A - инвариантное справа кольчо;

(3) все ииклические правые $A$-модули являются $m$-модулями.

ДоКАЗАТЕЛЬСТво. Импликация $(2) \Longrightarrow(1)$ следует из того, что $B=A B$ для каждого правого идеала $B$ инвариантного справа кольца $A$. Импликация $(1) \Longrightarrow(3)$ следует из леммы $1(1)$.

$(3) \Longrightarrow(2)$ Пусть $B$ - правый идеал кольца $A$. Так как циклический модуль $A_{A}-$ $m$-модуль, существует такой идеал $C$, что $B=A C=C$.

Лемма 3. Для нетерова справа кольца А равносильны условия:

(1) А дистрибутивно справа;

(2) в А все правые идеаль - произведения первичных двусторонних идеалов; 
(3) в А все правые идеаль - rm-идеаль;

(4) в А кажсдый первичный идеал - rm-идеал.

ДокАЗАТЕЛЬСТво. Эквивалентность условий $(1),(2)$ и (3) доказана в утверждениях $7.27(3)$ и 9.26 из [7]. Импликация (3) $\Longrightarrow(4)$ очевидна.

$(4) \Longrightarrow(2)$. Так как $A$ - первичньй идеал, по условию $A-r m$-идеал. По лемме $2 A$ инвариантно справа. Поэтому достаточно доказать, что в $A$ каждьй идеал - произведение первичных идеалов. Пусть $\mathscr{E}$ - множество всех собственных идеалов, не являющихся произведениями первичных идеалов. Допустим, что $\mathscr{E} \neq \varnothing$. Тогда $\mathscr{E}$ содержит максимальньй элемент $E$. Так как идеал $E$ не первичен, существуют такие идеалы $X$ и $Y$, что $X$ и $Y$ строго содержат $E$ и $X Y \subseteq E$. Так как $E$ - максимальный элемент в $\mathscr{E}$, то $X$ и $Y$ - произведения первичных идеалов, причем каждый из этих первичных идеалов строго содержит идеал $E$. Существуют такие первичные идеалы $P_{1}, \ldots, P_{n}$, что $X Y=P_{1} \cdots P_{n} \subseteq E$ и каждьй первичньй идеал $P_{i}$ строго содержит $E$. Так как каждый первичньй идеал - $r m$-идеал, то существуют такие идеалы $B_{1}, \ldots, B_{n}$, что $E=P_{i} B_{i} \subseteq B_{i}$ для любого $i$. Если хотя бы один из идеалов $B_{i}$ строго содержит максимальньй элемент $E$ множества $\mathscr{E}$, то $E$ - произведение первичных идеалов, поскольку $E=P_{i} B_{i}$. Поэтому $B_{i}=E$ для всех $i$. Тогда $E=P_{i} E$ для всех $i$. Поэтому $X Y E=P_{1} \cdots P_{n} E=P_{1} \cdots P_{n-1} E=\cdots=E \subseteq X Y \subseteq E$. Тогда $E=X Y=P_{1} \cdots P_{n}$ и $E$ - произведение первичных идеалов. Получили противоречие.

Лемма 4. Для модуля $M_{A}$ равносильны условия:

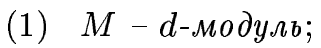

(2) для любих $x, y \in M$ существует $a \in A$, для которого $x a \in y A$ u $y(1-a) \in x A$;

(3) для любого $P \in \max \left(A_{A}\right)$ множсество $C_{P}(M)$ линейно упорядочено по включению;

(4) для любого $P \in \max \left(A_{A}\right)$ и любых $x, y \in M \quad P$-замыкания в $M$ модулей $x A$ и уА сравнимы по включению.

ДокаЗАтельство. (1) $\Longleftrightarrow(2)$ см. в [7, Proposition 1.17]. Импликация $(3) \Longrightarrow(4)$ очевидна.

$(4) \Longrightarrow(2)$. Допустим, что (2) невыполнено для некоторых $x, y \in M$. Тогда $(y A: x)+$ $(x A: y) \subseteq P \in \max \left(A_{A}\right)$. Пусть $X$ и $Y-P$-замыкания в $M$ модулей $x A$ и $y A$. Тогда $y \in Y \backslash X$ и $x \in X \backslash Y$. Это противоречит (4).

$(2) \Longrightarrow(3)$. Пусть $P \in \max \left(A_{A}\right), X, Y \in C_{P}(M), x \in X$ и $y \in Y$. По (2) существует $a \in A$, для которого $x a \in Y$ и $y(1-a) \in X$. Так как $X, Y \in C_{P}(M)$ и хотя бы один из элементов $a, 1-a$ не лежит в $P$, то либо $x \in Y$, либо $y \in X$. Поэтому $X$ и $Y$ сравнимы по включению.

ЛЕмма 5. Пусть $M-$ правый модуль над кольиом $A$, причем для любого $P \in$ $\max \left(A_{A}\right)$ множество $A \backslash P$ перестановочно справа и мультипликативно замкнуmo. Тогда

(1) для любых $N \in \operatorname{Lat}(M)$ и $P \in \max \left(A_{A}\right) \quad P$-замыкание $X$ модуля $N$ в $M$ является подмодулем в $M$;

(2) $M-d$-модуль $\Longleftrightarrow$ для любого $P \in \max \left(A_{A}\right)$ любые два $P$-замкнутых подмодуля модуля $M$ сравнимы по включению. 
ДокаЗАтельство. (1) Пусть $x, y \in X$ и $a \in A$. Надо доказать, что $x a, x+y \in X$. Сушествуют $s, s^{\prime} \in A \backslash P$, для которых $x s, y s^{\prime} \in N$. По условию $a t=s b$ и $s b^{\prime}=s^{\prime} t^{\prime} \in$ $A \backslash P$ для некоторых $t, t^{\prime} \in A \backslash P$ и $b, b^{\prime} \in A$. Тогда $(x a) t=(x s) b \in N$ и $(x+y) s^{\prime} t^{\prime}=$ $(x s) b^{\prime}+\left(y s^{\prime}\right) t^{\prime} \in N$. Поэтому $x a, x+y \in X$.

(2) Следует из (1) и леммы 4.

Лемма 6. Пусть $A-$ инвариантное справа кольцо. Тогда

(1) $B(1-b) \subseteq(1-b) B$ для кажсдого правого идеала $B$ кольиа $A$ и любого $b \in B$;

(2) если $B$ - идеал кольиа $A$ и $M$ - такой ииклический правый $A$-модуль, что $M=M B$, mo $A=B+r(M)$

(3) пусть $n$ - натуральное число $\geqslant 2, M-n$-порожсденный $A$-модуль с образуюшими $m_{1}, \ldots, m_{n}, \quad N=\sum_{i=1}^{n-1} m_{i} A$ и $B$ - такой идеал кольца $A$, что $M=M B ; \operatorname{moгда~} A=B+\left(N: m_{n} A\right) u N=N B$

(4) если $M$ - конечно порохденный правый $A$-модуль и $B$ - такой идеал кольиа $A$, что $M=M B$, mо $A=B+r(M)$

(5) если $M$ - конечно порожсденый правый $A$-модуль, то $M \neq M B$ для каждого собственного идеала $B$ кольиа $A$, содержащего $r(M)$;

(6) если $B$ - конечно порожденный идеал кольиа $A$ и $B=B^{2}$, то существует такой иентральный идемпотент е кольца $A$, что $B=e A$;

(7) если $M-$ - деал кольиа $A$, порожденный некоторым множеством идемпотентов, то $M$ - гт-идеал;

(8) если $M \in \bmod (A)$, то $M-d$-модуль $\Longleftrightarrow$ для любого $P \in \max \left(A_{A}\right)$ любие два $P$-замкнутых подмодуля модуля $M$ сравнимы по включению.

ДоказАтельство. (1) Пусть $b_{1} \in B$. Так как $A$ инвариантно справа, то $(1-b) A$ - идеал в $A$. Поэтому существует $a \in A$, для которого $b_{1}(1-b)=(1-b) a$. Тогда $a=(1-b) a+b a=b_{1}(1-b)+b a \in B$ и $B(1-b) \subseteq(1-b) B$.

(2) Пусть $M=m A$. Так как $A$ инвариантно справа, то $r(M)=r(m)$ и $m A=m A B=$ $m B$ поусловию. Поэтому $m(1-b)=0$ для некоторого $b \in B$. Тогда $A=B+r(m)=A+$ $r(M)$.

(3) Положим

$$
Y= \begin{cases}0, & \text { если } n=2, \\ \sum_{i=2}^{n-1} m_{i} A, & \text { если } n \geqslant 3 .\end{cases}
$$

Для каждого $m_{i} m_{i} \in M=M B=\sum_{j=1}^{n} m_{j} B$ и существует $b_{i} \in B$, для которого $m_{i}\left(1-b_{i}\right) \in \sum_{j \neq i} m_{j} B$. Тогда $m_{n} A\left(1-b_{n}\right) \subseteq N B=m_{1} B+Y B$ и $m_{1} A\left(1-b_{1}\right) \in$ $Y B+m_{n} B$, поскольку $A$ инвариантно справа; кроме того, $B\left(1-b_{1}\right) \subseteq\left(1-b_{1}\right) B$ по (1). Поэтому $A=B+\left(N: m_{n} A\right)$ и $m_{1} A\left(1-b_{1}\right)\left(1-b_{n}\right) \subseteq\left(Y B+m_{n} B\right)\left(1-b_{n}\right) \subseteq Y B+$ $m_{n} B\left(1-b_{n}\right) \subseteq Y B+m_{n}\left(1-b_{n}\right) B \subseteq Y B+m_{1} B=N B$. Тогда существует $b^{*} \in B$, для которого $m_{1}\left(1-b_{1}\right)\left(1-b_{n}\right)-m_{1} b^{*} \in Y B$ и $m_{1}\left[\left(1-b_{1}\right)\left(1-b_{n}\right)-b^{*}\right] \in Y B$. Положим $b=1-\left[\left(1-b_{1}\right)\left(1-b_{n}\right)-b^{*}\right] \in B$. Тогда $m_{1}=m_{1} b+m_{1}(1-b)=m_{1} b+m_{1}\left[\left(1-b_{1}\right)(1-\right.$ $\left.\left.b_{n}\right)-b^{*}\right] \in m_{1} B+Y B=N B$. Поэтому $m_{1} A \subseteq N B$. Аналогично можно доказать, что $m_{i} A \subseteq N B$ при $i=2, \ldots, n-1$. Поэтому $N=N B$.

(4) Проведем индукцию по $n$. При $n=1$ утверждение следует из (2). Допустим, что $n \geqslant 2$ и утверждение верно для всех натуральных чисел, меньших $n$. Пусть $N=$ $\sum_{i=1}^{n-1} m_{i} A$. По (3) $A=B+\left(N: m_{n} A\right)$ и $N=N B$. По предположению индукции $A=B+r(N)$. Тогда $M\left(N: m_{n} A\right) r(N)=\left(N+m_{n} A\right)\left(N: m_{n} A\right) r(N)=N(N$ : 
$\left.m_{n} A\right) r(N)+m_{n} A\left(N: m_{n} A\right) r(N) \subseteq N r(N)=0$. Поэтому $\left(N: m_{n} A\right) r(N) \subseteq r(M)$ и $A=A \cdot A=\left(B+\left(N: m_{n} A\right)\right)(B+r(N)) \subseteq B+\left(N: m_{n} A\right) r(N) \subseteq B+r(M) \subseteq A$.

(5) Утверждение следует из (4).

(6) Так как $B$ - конечно порожденньй идеал инвариантного справа кольца $A$, то $B$ конечно порожденньй правьй идеал. По (4) $A=B+r(B)$. Поэтому существует $e \in B$, для которого $B(1-e)=0$. В частности, $e(1-e)=0$ и $e=e^{2}$. Так как $e A$ и $(1-e) A-$ идеалы инвариантного справа кольца $A$, идемпотент $е$ централен.

(7) Пусть $M=\sum_{i \in I} e_{i} A\left(e_{i}^{2}=e_{i}\right)$ и $B$-правый идеал кольца $A$, содержашийся в $M$. Так как $A$ инвариантно справа, то $M B \subseteq B$. Поэтому достаточно доказать, что $B \subseteq$ $M B$. Пусть $b \in B$. Существует такое конечное подмножество $\left\{e_{1}, \ldots, e_{n}\right\}$ в $\left\{e_{i}\right\}_{i \in I}$, что $b=\sum_{i=1}^{n} e_{i} a_{i}$ для некоторых $a_{1}, \ldots, a_{n} \in A$. Так как $A$ инвариантно справа, все идемпотенты $e_{i}$ центральны. Тогда $\left(1-e_{1}\right) \cdot \ldots \cdot\left(1-e_{n}\right) b=0$ и $b=\left(1-\left(1-e_{1}\right) \cdot \ldots \cdot\right.$ $\left.\left(1-e_{n}\right)\right) b \in\left(\sum_{i=1}^{n} e_{i} A\right) b \subseteq M B$. Поэтому $B \subseteq M B$.

(8) Так как $A$ инвариантно справа, для любого $P \in \max \left(A_{A}\right)$ множество $A \backslash P$ перестановочно справа и мультипликативно замкнуто. Теперь применяем лемму $5(2)$.

Лемма 7. Пусть $M-$ правый модуль над дистрибутивным справа инвариантным справа кольиом $A$. Тогда

(1) $M B \cap M C=M(B \cap C)$ для любых правых идеалов $B$ и $C$ из $A$;

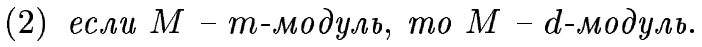

ДокАЗАТЕЛЬСТВо. (1) доказано в $[7,7.27(4)]$, а (2) следует из (1) и леммы 1(6).

Лемма 8. Для кольиа А равносильны следующие условия:

(1) A - дистрибутивное справа нетерово справа кольцо и каждое факторкольчо кольца $A$ является конечным прямым произведением областей $и$ артиновых справа иепных справа колеи;

(2) A - дистрибутивное справа нетерово справа кольио и для любого его фактор-кольиа $R$ с первичным радикалом $N$ верно, что либо $R$ - артиново справа кольчо, либо $N$ не является существенным правым идеалом кольu $R$;

(3) $A$ - конечное прямое произведение артиновых справа иепных справа колеи и таких инвариантных справа областей $D_{i}$, что для каждого $D_{i}$ любое собственное фактор-кольчо области $D_{i}$ является конечным прямым произведением артиновых справа иепных справа колец.

ДокАЗАТЕЛЬСТво. Импликации $(3) \Longrightarrow(1)$ и $(1) \Longrightarrow(2)$ очевидны. Импликация $(2) \Longrightarrow(3)$ доказана в $[7,9.27(4)]$.

Лемма 9. Пусть $A$ - дистрибутивное справа нетерово справа неразложимое кольио. Допустим, что $A$ не является областью и $A$ не является артиновым справа чепным справа кольцом. Тогда А имеет такой ненулевой вполне первичный нильпотентный идеал $X$ и максимальный идеал $Y$, что $X=Y X \subsetneq Y$, $X Y \in \max (X) u X Y \neq Y X$.

ДокАЗАтЕльство. По лемме $3 A$ инвариантно справа. В $[7,9.20(1)]$ доказано, что $A$ имеет такой ненулевой вполне первичньй нильпотентньй идеал $X$, что $X=y X$ для каждого $y \in A \backslash X$. Допустим, что $X \notin \max \left({ }_{A} A_{A}\right)$. Ненулевой модуль $X_{A}$ имеет максимальньй подмодуль $K$. Обозначим через $Y$ идеал $r(X / K)$ кольца $A$. Так как $A$ 
инвариантно справа и $A$-модуль $X / K$ прост, то $Y \in \max \left({ }_{A} A_{A}\right)$. Тогда $X Y \subseteq K \neq X$. Так как $X \notin \max \left({ }_{A} A_{A}\right)$, то $Y \nsubseteq X$. Существует $y \in Y \backslash X$. Тогда $X=y X \subseteq Y X \subseteq X$. Поэтому $X=Y X$. Допустим, что $X$ - максимальный идеал инвариантного справа кольца $A$. Тогда $A$ - нетерово справа полупримарное локальное кольцо. Поэтому $A$ apтиново справа с единственным собственным первичным идеалом $J$. Из леммы 3 следует, что $A$ - цепное справа кольцо. Получили противоречие.

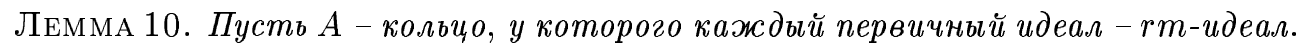
Tогда

(1) если $P$ и $Q$ - первичные идеалы кольиа $A$ и $P \nsubseteq Q$, mо $P \cap Q=P Q$;

(2) если $P$ и $Q$ - первичные идеаль кольца $A$ и $P \neq Q$, то либо $Q=P Q \subsetneq P$, либо $P=Q P \subsetneq Q$, либо $P Q=Q P=P \cap Q$.

ДоказАТельство. (1) Так как $P$ и $Q-r m$-идеалы, то $P \cap Q=P B=Q C$ для некоторых идеалов $B$ и $C$. Так как $P \nsubseteq Q$ и $P B$ лежит в первичном идеале $Q$, то $B \subseteq Q$. Поэтому $P \cap Q=P B \subseteq P Q \subseteq P \cap Q$. Тогда $P \cap Q=P Q$.

(2) Если $P$ и $Q$ не сравнимы по включению, то по (1) $P Q=Q P=P \cap Q$. Поэтому можно считать, что $Q \subsetneq P$. Тогда $P \nsubseteq Q$ и $Q=P \cap Q=P Q$ по (1).

Лемма 11. Пусть $A$ - инвариантное кольцо, $X$ - его ненулевой идеал, $Q$ классическое кольио частных кольиа $A$. Тогда

(1) если $X-m$-идеал, содержсащий $x \in S(A)$, то идеал $X$ обратим в $Q u$ является конечно порохсденным проективным правым (левым) идеалом;

(2) идеал $X$ обратим в $Q \Longleftrightarrow X-m$-идеал, содержащий $x \in S(A)$;

(3) если $X-r m$-идеал, то $P X \subsetneq X$ для некоторого $P \in \max \left(A_{A}\right)$;

(4) если $X$ - первичный гт-идеал $u X \subseteq J(A)$, то $A$ - локальное кольцо и $J(A)=X=x A=A x$ для некоторого $x \in X$.

ДоказАтельство. (1) Существуют такие идеалы $B$ и $C$, что $X B=x A=A x=$ $C X$. Поэтому $X\left(B x^{-1}\right)=A=\left(x^{-1} C\right) X$. Тогда (1) следует из утверждения $7.35(2)$ В $[7]$.

(2) По (1) достаточно доказать $\Longrightarrow$. По условию $X X^{*}=X^{*} X=A$ для некоторого подбимодуля $X^{*}$ в ${ }_{A} Q_{A}$ и $1=\sum_{i=1}^{n} m_{i} q_{i}$ для некоторых $m_{1}, \ldots, m_{n} \in X$ и $q_{1}, \ldots, q_{n} \in$ $X^{*}$. Существуют такие $a_{1}, \ldots, a_{n} \in A$ и $x \in S(A)$, что $q_{i}=a_{i} x^{-1}, i=1, \ldots, n$. Поэтому $x=\sum_{i=1}^{n} m_{i} q_{i} x=\sum_{i=1}^{n} m_{i} a_{i} \in X$. Пусть $Y$-идеал инвариантного кольца $A$, лежащий в $X, B=X^{*} Y \subseteq A$ и $C=Y X^{*} \subseteq A$. Тогда $Y=X X^{*} Y=X B=Y X^{*} X=$ $C Y$ и $X-m$-идеал.

(3) Пусть $0 \neq y \in X$ и $Y=y A=A y$. Тогда $P Y \neq Y$ для некоторого $P \in \max \left({ }_{A} A\right)=$ $\max \left(A_{A}\right)$. Так как $X-r m$-идеал, то $Y=X B$ для некоторого идеала $B$. Если $X=P X$, то $Y=X B=P X B=P Y \neq Y$ и получаем противоречие.

(4) По (3) $P X \subsetneq X$ для некоторого $P \in \max \left(A_{A}\right)$. Если $P \nsubseteq X$, то по лемме $10(1)$ $X \subseteq P \cap X=P X \subsetneq X$ и получаем противоречие. Поэтому $P \subseteq X \subseteq J(A) \subseteq P$, $X=P=J(A)$ - единственный максимальньй правьй идеал и $A$ локально. По (3) существует $x \in X \backslash X^{2}$. Так как $X-r m$-идеал и $x \notin X^{2}$, то $x A=X B$ для некоторого идеала $B \nsubseteq X$. Тогда $B=A$ и $X=X B=x A=A x$. 
Лемма 12. Пусть $A$ - инвариантное кольио с ненулевым первичным радика-

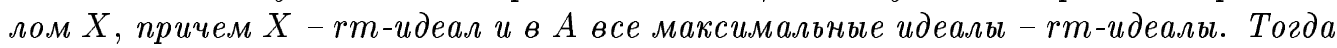

(1) либо $A$ - иепное артиново кольчо главных идеалов, либо $A / X=P / X \times Q / X$, где $P / X$ - ненулевое редуиированное кольио и $Q / X$ - тело;

(2) если $A / X$ - неразложимое кольцо, то $A$ - чепное артиново кольцо главных идеалов;

(3) если $A$ - неразложимое кольио, то $A$ - чепное артиново кольио главных идеалов.

ДокАЗАтЕльство. (1) Так как $A / X$ - инвариантное полупервичное кольцо, то $A / X$ редуцировано. По лемме $11(3)$ существует $P \in \max \left(A_{A}\right)$, для которого $P X \subsetneq X \subseteq$ $J(A) \subseteq P$. Так как $P-r m$-идеал, то $X=P Q \subseteq P \cap Q$ для некоторого идеала $Q$. Тогда $(P \cap Q)^{2}$ содержится в полупервичном идеале $X$. Поэтому $P \cap Q \subseteq X \subseteq P \cap Q$. Тогда $X=P \cap Q \subsetneq Q$, поскольку $P X \subsetneq X$. Допустим, что $X=P$. По лемме 11(4) $A$ локально и $J(A)=X=x A=A x$ для некоторого $x \in X$. Кроме того, $x^{n}=0$ для некоторого $n$. Тогда $X^{n}=0$ и множество всех собственных правых (левых) идеалов кольца $A$ совпадает со множеством степеней идеала $X$ и $A$ - цепное артиново кольцо главных идеалов. Остается случай, когда $X \subsetneq P$ и $X=P Q=P \cap Q \subsetneq Q$. Тогда $Q$ не лежит в максимальном идеале $P$. Поэтому $A=P+Q$ и $A / X=P / X \times Q / X$, где ненулевое прямое слагаемое $P / X$ редуцированного кольца $A / X$ является редуцированным кольцом и $Q / X$ - тело, поскольку $Q / X \cong(A / X) /(P / X) \cong A / P$.

(2) Следует из (1).

(3) Так как $A$ неразложимо и инвариантно, $A$ не имеет нетривиальных идемпотентов. Кроме того, все нетривиальные идемпотенты кольца $A / X$ поднимаются до нетривиальных идемпотентов в $A$, поскольку $X$-нильидеал. Поэтому $A / X$-неразложимоекольцо. По (2) $A$ - цепное артиново кольцо главных идеалов.

Лемма 13. Пусть $A$ - инвариантное справа кольио. Если $A$ не является нетеровым справа, то $A$ имеет такой собственный вполне первичный идеал $B$, что $B$ не конечно порожден, а каждый идеал кольца $A$, строго содержсащий $B$, конечно порохсден. Следовательно, если все вполне первичные идеаль кольиа $А$ конечно порождены, то А нетерово справа.

ДокАЗАТЕЛЬСТво. Пусть $\left\{B_{i}\right\}_{i \in I}-$ непустое множество всех собственньх идеалов, не являющихся конечно порожденными. Так как объединение любой цепи элементов множества $\left\{B_{i}\right\}_{i \in I}$ содержится в этом множестве, по лемме Цорна $\left\{B_{i}\right\}_{i \in I}$ содержит некоторый максимальньй элемент $B$. Пусть $x \in A \backslash B$ и $Y=(B: x)$. Достаточно доказать, что $Y \subseteq B$. Допустим противное. Тогда $x y \in B$ для некоторого $y \in Y \backslash B$. Так как $x B \subseteq B$ и $y \in Y \backslash B$, идеалы $Y$ и $x A+B$ строго содержат $B$. Поэтому существуют такие $y_{1}, \ldots, y_{m} \in Y$ и $b_{1}, \ldots, b_{n} \in B$, что $Y=\sum_{i=1}^{m} y_{i} A$ и $x A+B=x A+\sum_{j=1}^{n} b_{j} A$. Тогда $B \cap x A=x Y=\sum_{i=1}^{m}\left(x y_{i}\right) A$ и $B=B \cap(x A+B)=B \cap\left(x A+\sum_{j=1}^{n} b_{j} A\right)=B \cap$ $x A+\sum_{j=1}^{n} b_{j} A=\sum_{i=1}^{m}\left(x y_{i}\right) A+\sum_{j=1}^{n} b_{j} A$. Поэтому $B$ конечно порожден. Получили противоречие.

Лемма 14. (1) Пусть все полупервичные идеаль кольиа $A$ являются rm-идеалами, причем либо А равномерно, либо А обладает только одним минимальным первичным идеалом. Тогда $A$ - либо инвариантная область, либо инвариантное иепное артиново кольцо главных идеалов. 
(2) Если $X$ - первичный идеал кольца $A$ и все вполне первичные идеаль кольиа $A$ являются $m$-идеалами, то $A / X$ - инвариантная нетерова область, у которой все ненулевые идеалы обратимы.

ДокАЗАТЕЛьСТво. По лемме 2 кольцо $A$ инвариантно.

(1) Пусть $A$ - не область и $X$ - первичный радикал кольца $A$. Так как $X$ - полупервичный идеал, то $X-r m$-идеал по условию. Допустим, что $A$ равномерно. Так как $A-$ не область и любое инвариантное равномерное полупервичное кольцо является областью, то $X \neq 0$. По лемме $12(3) A$-цепное артиново кольцо главных идеалов. Допустим, что $A$ обладает только одним минимальным первичньм идеалом. Тогда $X$-первичный идеал. По лемме 12(2) $A$ - цепное артиново кольцо главных идеалов.

(2) Инвариантное первичное кольцо $A / X$ является областью, у которой все вполне первичные идеалы - $m$-идеалы. По лемме 11(1) все ненулевые вполне первичные идеалы области $A / X$ конечно порождены и обратимы. По лемме $13 A / X$ - нетерова область. По лемме 3 каждьй ненулевой идеал $B / X$ области $A / X$ - произведение ненулевых вполне первичных обратимых идеалов. Поэтому $B / X$ обратим.

ЛЕмма 15. Пусть в кольце А все полупервичные идеалы являются Тогда

(1) если $X_{1}, \ldots, X_{n}$ - такие идеаль кольиа $A$, что все фактор-кольиа $A / X_{i}$ нетеровы справа, то $A /\left(\bigcap_{i=1}^{n} X_{i}\right)$ - конечное прямое произведение инвариантных чепных артиновых колец главных идеалов и инвариантных нетеровых областей, у которых все ненулевые идеалы обратимы;

(2) если либо $A$ обладает только конечным числом минимальных первичных идеалов, либо $A$ не содержит бесконечных прямых сумм ненулевых идеалов, то $A$ - конечное прямое произведение инвариантных иепных артиновых колец главных идеалов и инвариантных нетеровых областей, у которых все ненулевые идеаль обратимы.

ДокАЗАТЕЛьСТво. По лемме 2 кольцо $A$ инвариантно.

(1) Пусть $\bar{A}=A /\left(\bigcap_{i=1}^{n} X_{i}\right)$. Так как все модули $\left(A / X_{i}\right)_{A}$ нетеровы, то $\bar{A}_{A}$ - нетеров модуль. Поэтому $\bar{A}$-нетерово справа кольцо, у которого все полупервичные идеалы являются $m$-идеалами. Из леммы 9 следует, что $\bar{A}=\bar{A}_{1} \times \cdots \times \bar{A}_{n}$, где все кольца $\bar{A}_{i}$ почти целостны. Так как каждое кольцо $\bar{A}_{i}$ обладает единственным минимальным первичным идеалом, по лемме $14 \bar{A}_{i}$ - либо инвариантное цепное артиново кольцо главньх идеалов, либо инвариантная нетерова область, у которой все ненулевые идеалы обратимы.

(2) Допустим, что инвариантное кольцо $A$ не содержит бесконечных прямых сумм ненулевых идеалов. Тогда $A$ содержит такие идеалы $B_{1}, \ldots, B_{n}$, что все фактор-кольца $A / B_{i}$ равномерны и $\bigcap_{i=1}^{n} B_{i}=0$. По лемме 14 все кольца $A / B_{i}$ нетеровы. Теперь $(2)$ следует из (1). Допустим, что множество всех минимальных первичных идеалов кольца $A$ конечно и равно $\left\{X_{i}\right\}_{i=1}^{n}$. Пусть $X=\bigcap_{i=1}^{n} X_{i}$ - первичньй радикал кольца $A$. По лемме $14(2)$ все фактор-кольца $A / X_{i}$ нетеровы. По (1) кольцо $A / X$ нетерово. Из леммы 9 следует, что $A / X$ - конечное прямое произведение почти целостных колец. Кроме того, $A / X$ полупервично и $X$ - нильидеал. Поэтому $A / X$ - конечное прямое произведение областей и $A=A_{1} \times \cdots \times A_{n}$, где первичньй радикал каждого кольца $A_{i}$ является первичным идеалом в $A_{i}$. Теперь (2) следует из леммы 14.

ОКОНЧАНИЕ ДОКАЗАТЕЛЬСТВА ТЕОРЕМЫ А. ЭквиваЛентность условий (1)-(3) следует из леммы 3 . Импликация $(1) \Longrightarrow(5)$ следует из леммы 2 и леммы 6(5). Импликация 
$(5) \Longrightarrow(1)$ следует из леммы $1(3)$. Импликация $(4) \Longrightarrow(3)$ следует из леммы 2 . Импликация $((1)+(3)) \Longrightarrow(4)$ следует из леммы $1(5)$, леммы 2 и леммы $7(2)$.

ПРЕДЛОЖЕНИЕ 16. Пусть кольцо $A$ либо не содержит бесконечных прямых сумм ненулевых идеалов, либо имеет лишь конечное число минимальных первичных идеалов. Равносильны условия:

(1) $A$ - нетерово дистрибутивное кольио;

(2) в кольие $A$ все правые идеаль - rт-идеаль и все левые идеаль - lm-идеаль;

(3) в кольие $A$ все полупервичные идеаль -

(4) $A$ - конечное прямое произведение инвариантных артиновых локалыных колеи, главных идеалов и инвариантных нетеровых областей, у которых все ненулевые идеалы являются обратимыми.

ДокАЗАТЕльство. Эквивалентность $(1) \Longleftrightarrow(2)$ следует из теоремы А. Импликация $(2) \Longrightarrow(3)$ очевидна. Импликация $(3) \Longrightarrow(4)$ следует из леммы $15(2)$. Импликация $(4) \Longrightarrow(2)$ следует из лемм 11(2) и 2 .

ОКОНЧАНИЕ ДОКАЗАТЕЛЬСТВА ТЕОРЕМЫ В. (1) Импликация посредственно. Докажем $\Longrightarrow$ Так как каждое фактор-кольцо дистрибутивного справа нетерова справа кольца с коммутативным умножением первичных идеалов обладает теми же свойствами и является конечным прямым произведением неразложимых колец, по лемме 8 достаточно доказать, что каждый неразложимьй прямой сомножитель $\bar{A}$ кольца $A$ является либо областью, либо артиновьм справа цепным справа кольцом. Это следует из леммы 9.

(2) Следует из предложения 16.

(3) В утверждении 7.7(1) из [7] доказано, что для любого максимального правого идеала $P$ дистрибутивного справа кольца $A$ множество $A \backslash P$ перестановочно справа и мультипликативно замкнуто. Теперь применяем лемму $5(2)$.

\section{СПИСОК ЦИТИРОВАННОЙ ЛИТЕРАТУРЫ}

[1] Smith W. W. Projective ideals of finite type // Canad. J. Math. 1969. V. 21. P. 1057-1061.

[2] Barnard A. Multiplication modules // J. Algebra. 1981. V. 71. № 1. P. 174-178.

[3] El-Bast Z. A., Smith P. F. Multiplication modules // Comm. Algebra. 1988. V. 16. № 4. P. 755-779.

[4] Anderson D. D. Some remarks on multiplication ideals // Math. Japonica. 1980. V. 4. № 4. P. 463-469.

[5] Smith P. F. Some remarks on multiplication modules // Arch. Math. 1988. V. 50. №3. P. 223-235.

[6] Smith P. F. Multiplication modules and projective modules // Period. Math. Hungar. 1994. V. 29. № 2. P. 163-168.

[7] Tuganbaev A. A. Semidistributive Modules and Rings. Dordrecht-Boston-London: Kluwer Academic Publ., 1998.

[8] Tuganbaev A. A. Distributive Modules and Related Topics. Amsterdam: Gordon and Breach Science Publ., 1999. 